\title{
Criança com estoma nos estudos de Pós-Graduação Stricto Sensu em Enfermagem: destacando aspectos epistemológicos
}

\author{
Child with stoma in Nursing post-graduation studies: epistemological aspects
}

Niño con estoma en estudios de Postgrado en Enfermería: destacando aspectos epistemológicos

Thiago Privado da Silva', Italo Rodolfo Silva", Laura Johanson da Silva'", Maria José Carvalho Ferreiralv, Marlea Crescêncio Chagas" , Carina Bulcão Pinto ${ }^{\mathrm{VI}}$

\begin{abstract}
RESUMO
Objetivo: analisar as dissertações e teses defendidas nos cursos de Pós-Graduação Stricto Sensu em Enfermagem, no Brasil, relativas ao tema criança com estoma, destacando seus aspectos epistemológicos. Método: estudo documental, descritivo, considerando o recorte temporal entre 2009 e 2019. Utilizou-se a Metodologia de Categorização Epistemológica para a Pesquisa na Enfermagem. Resultados: selecionaram-se doze estudos: dez dissertações de mestrado e duas teses de doutorado. Nota-se o predomínio de estudos na área/campo epistêmico assistencial, na linha de pesquisa: Processo de Cuidar em Saúde e Enfermagem. Identificou-se um maior interesse de estudos no contexto domiciliar e ambulatorial. Há predomínio da abordagem qualitativa e uso da entrevista para coleta de dados. Conclusão: os aspectos epistemológicos destacados possibilitam afirmar que as dissertações e teses relacionadas ao tema estão predominantemente orientadas para um pragmatismo epistemológico da assistência de enfermagem à criança com estoma e sua família, com objetos científicos delimitados para alcançar a compreensão desse fenômeno.
\end{abstract}

Descritores: Pesquisa; Educação de Pós-graduação; Enfermagem Pediátrica; Estomas Cirúrgicos.

\begin{abstract}
Objective: to examine postgraduate Nursing dissertations and theses defended in Brazil on the subject of children with stoma, highlighting their epistemological aspects. Method: this descriptive study considered studies published between 2009 and 2019 using the Epistemological Categorization methodology for research in Nursing. Results: twelve studies were selected: ten master's dissertations and two doctoral theses. The predominant epistemic area/field was "care", in the research line: "care process in health, and nursing". Studies were found to show greater interest in the home and outpatient context. The qualitative approach and data collection by interview predominated. Conclusion: from the epistemological aspects highlighted, it can be said that the dissertations and theses on the subject of children with stomata were predominantly oriented towards an epistemological pragmatism in nursing care for children with stoma and their families, with scientific objects delimited to achieve an understanding of this phenomenon.
\end{abstract}

Descriptors: Research; Graduate; Pediatric Nursing; Surgical Stomas; Education.

\section{RESUMEN}

Objetivo: analizar las disertaciones y tesis de posgrado en Enfermería defendidas en Brazil sobre el tema de niños con estoma, destacando sus aspectos epistemológicos. Método: este estudio descriptivo consideró estudios publicados entre 2009 y 2019 utilizando la metodología de Categorización Epistemológica para la investigación en Enfermería. Resultados: se seleccionaron doce estudios: diez tesis de maestría y dos tesis doctorales. El área / campo epistémico predominante fue el "cuidado", en la línea de investigación: "proceso de cuidado en salud y enfermería". Se encontró que los estudios muestran un mayor interés en el contexto domiciliario y ambulatorio. Predominó el enfoque cualitativo y la recolección de datos por entrevista. Conclusión: a partir de los aspectos epistemológicos destacados, se puede decir que las disertaciones y tesis sobre el tema de los niños con estoma estuvieron orientadas predominantemente hacia un pragmatismo epistemológico en la atención de enfermería al niño con estoma y sus familias, con objetos científicos delimitados para lograr un entendimiento. de este fenómeno.

Descriptores: Investigación; Educación de Postgrado; Enfermería Pediátrica; Estomas Quirúrgicos.

\section{INTRODUÇÃO}

A produção de conhecimento sobre o manejo de estomas no contexto assistencial de enfermagem apresenta-se como uma prioridade, posto que várias condições de saúde podem demandar a confecção de um estoma, solicitando do enfermeiro conhecimentos e habilidades para um adequado gerenciamento do cuidado. Assim, a formação de especialistas, mestres e doutores com expertise no tema faz-se necessária para fomentar práticas de cuidados de enfermagem seguras e eficazes.

'Enfermeiro. Doutor. Professor Assistente. Universidade Federal do Rio de Janeiro Campus Macaé Professor Aloísio Teixeira. Brasil. E-mail: thiagopsilva87@gmail.com. ORCID: http://orcid.org/0000-0002-7744-8319

"Enfermeiro. Doutor. Professor Adjunto. Universidade Federal do Rio de Janeiro Campus Macaé Professor Aloísio Teixeira. Brasil. E-mail: enf.italo@hotmail.com. ORCID: http://orcid.org/0000-0002-2882-1877

I"Enfermeira. Doutora. Professora Adjunto. Universidade Federal do Estado do Rio de Janeiro. Brasil. E-mail: lauraenfaunirio@gmail.com. ORCID: http://orcid.org/0000-0002-4439-9346 IVEnfermeira. Mestre. Aluna do curso de doutorado. Universidade Federal do Rio de Janeiro. Brasil. E-mail: maria.jcarvalho@live.com. ORCID: http://orcid.org/0000-0002-6165-8709 vEnfermeira. Doutora. Professora Associada. Universidade Federal do Rio de Janeiro. Rio de Janeiro, Brasil. E-mail: marleachagas@gmail.com. ORCID: https://orcid.org/0000-0002-6122-7300 V'Enfermeira. Mestre em Enfermagem. Professora Assistente. Universidade Federal do Rio de Janeiro Campus Macaé Professor Aloísio Teixeira. Brasil. E-mail: cacabulcao@gmail.com. ORCID: http://orcid.org/0000-0001-9862-6113 
No contexto do exercício profissional, a Estomaterapia é uma especialidade da Enfermagem cujas atividades de ensino, pesquisa e assistência são orientadas para o atendimento de pessoas com estomas, feridas, fístulas, drenos, cateteres e incontinências ${ }^{1}$. Encontra-se em franca expansão, oferecendo possibilidades de trabalho no âmbito da assistência, administração, vendas, ensino, pesquisa, assessoria e consultoria ${ }^{2-3}$.

No âmbito da Enfermagem em Estomaterapia, este estudo aborda o estoma, o qual pode ser compreendido como a exteriorização de um órgão interno para a superfície corporal. Dependendo da sua finalidade é subdividido em grupos como estomas de eliminação que podem ser urinários (urostomia) ou intestinais (colostomia e ileostomia); estomas de alimentação (gastrostomia e jejunostomia); estomas de ventilação (traqueostomia); e estomas especiais (esofagostomia, pleurostomia, peritoneostomia etc.) ${ }^{4}$.

Na saúde da criança, dentre as principais condições que podem levar à confeç̧ão de um estoma, estão ${ }^{5,6}$ megacólon congênito, imperfuração anal, insuficiência respiratória aguda, doença do refluxo gastroesofágico, perfuração intestinal, atresia do esôfago, bexiga neurogênica, estenose uretral, laringotraqueomalácia, peritonite aguda, estenose subglótica, dentre outras.

Nesse ínterim, ressalta-se a vulnerabilidade da criança com estoma em todos os contextos de cuidado, haja vista que a sua condição de saúde demanda dos serviços sociais e de saúde políticas públicas, rede de apoio de diversas naturezas, além de acompanhamento e assistência especializada ${ }^{5}$, inclusive para a família.

Dada a complexidade que envolve o cuidado à criança com estoma, é notória a relevância de pesquisas sobre o tema no âmbito da Pós-Graduação Lato Sensu e Stricto Sensu em Enfermagem. A esse respeito, nota-se um crescimento expressivo no número de cursos de Pós-Graduação Lato Sensu em Enfermagem, em Estomaterapia, no Brasil7.

Nessa direção, pesquisa revelou um aumento no número de pesquisas sobre estomas na esfera dos Programas de Pós-Graduação Stricto Sensu em Enfermagem, no Brasil ${ }^{8}$. Contudo, autores ${ }^{6,9}$ acrescentam a necessidade de aprofundar as pesquisas sobre o tema quando se trata do contexto pediátrico. Assim, questiona-se: como se caracterizam as dissertações e teses defendidas nos cursos de Pós-Graduação em Enfermagem, no Brasil, relativas ao tema criança com estoma? Quais aspectos epistemológicos dessa produção científica podem ser destacados?

A relevância deste estudo repousa na possibilidade de apontar tendências, lacunas e novos direcionamentos para o fortalecimento do conhecimento sobre o tema em tela. Ademais, a partir da Metodologia de Categorização Epistemológica para a Pesquisa na Enfermagem, será possível compreender a internalidade epistemológica da Enfermagem no que tange à produção de conhecimento sobre o tema.

Portanto, definiu-se como objetivo analisar as dissertações e teses defendidas nos cursos de Pós-Graduação em Enfermagem, no Brasil, relativas ao tema criança com estoma, destacando seus aspectos epistemológicos.

\section{MÉTODO}

Estudo documental, descritivo, utilizando como fontes para o levantamento de dados o Banco de Teses da Coordenação de Aperfeiçoamento de Pessoal de Nível Superior (CAPES) e o Catálogo de Teses e Dissertações do Centro de Estudos e Pesquisas em Enfermagem (CEPEn) da Associação Brasileira de Enfermagem (ABEn Nacional).

A busca de dados foi realizada em janeiro e fevereiro de 2020, considerando o seguinte critério de inclusão: dissertações e teses defendidas nos cursos de Pós-Graduação em Enfermagem cujo tema contemple a criança com estoma, entre os anos de 2009 e 2019, com resumos e/ou textos do material disponíveis na íntegra nos bancos de dados. Excluíram-se estudos duplicados, bem como os provenientes de Programas de Pós-Graduação Stricto Sensu de áreas afins.

Utilizou-se com marco inicial o ano de divulgação da Portaria de no 400, publicada em 16 de novembro de 2009, na qual estão delineadas as Diretrizes Nacionais para a Atenção à Saúde das Pessoas Ostomizadas, no âmbito do Sistema Único de Saúde - SUS, fato que reforçou no país a necessidade de oferecer à pessoa estomizada atenção integral e especializada a sua saúde.

Para o direcionamento da busca no Banco de Teses da CAPES, utilizaram-se as seguintes palavras-chave: criança, estoma, estomia, ostomia e ostoma. Adotaram-se as seguintes estratégias de combinação: Criança [AND] Estoma, Criança [AND] Estomia, Criança [AND] Ostomia e Criança [AND] Ostoma.

A estratégia de busca "Criança [AND] Estoma" possibilitou a identificação de 14 estudos. Destes, seis não tratavam de cursos de Pós-Graduação em Enfermagem e um não apresentava o texto disponível na Plataforma Sucupira, restando sete estudos para esta pesquisa. A combinação "Criança [AND] Estomia" culminou em 13 estudos, dos quais seis estavam duplicados e três não tratavam de cursos de Pós-Graduação em Enfermagem, culminando em quatro estudos para esta pesquisa. A estratégia de busca "Criança [AND] Ostomia" resultou em três estudos, todos pertencentes a cursos de Pós-Graduação de outras áreas da saúde. Por último, a combinação "Criança [AND] Ostoma” não apresentou 
nenhuma produção científica. Assim, a busca no Banco de Teses da CAPES possibilitou a seleção de 11 estudos que atenderam ao critério de inclusão definido.

A busca no Catálogo de Teses e Dissertações do CEPEn não seguiu a mesma estratégia de busca utilizada no Banco de Teses da CAPES, haja vista que esse banco de dados organiza seu conteúdo por volumes e ano, desse modo não possui a ferramenta para a busca com palavras-chave. Assim sendo, a estratégia definida foi realizar o download dos volumes, considerando o recorte temporal estabelecido e, em seguida, proceder à leitura dos títulos e resumos.

Logo, a busca foi iniciada com o volume de número 28, o qual contém estudos referentes ao ano de 2009. Nesse volume, apenas um estudo foi selecionado. No volume 29 , encontraram-se dois estudos sobre estomas, os quais não atenderam ao critério de inclusão. No volume 30, identificaram-se cinco estudos sobre o tema, porém quatro referentes à saúde do adulto e um proveniente de curso de Pós-Graduação Stricto Sensu de áreas afins. No volume 31, também foram identificados cinco estudos sobre o tema, sendo quatro referentes à saúde do adulto e um, à saúde do idoso. No volume 32, identificou-se um total de quatro estudos, dois atenderam ao critério de inclusão definido, porém ao considerar o critério de duplicação, os mesmos foram excluídos; os outros dois foram desenvolvidos no âmbito da saúde do adulto. No volume 33, os seis estudos sobre estoma contemplavam a saúde do adulto. Por último, no volume 34 , identificaram-se três estudos sobre o tema, todos referentes à saúde do adulto. Portanto, no Catálogo de Teses e Dissertações do CEPEn, selecionou-se apenas um estudo.

Desse modo, para a constituição do corpus deste estudo, selecionaram-se 12 produções científicas que atenderam ao critério de inclusão definido. Um instrumento de elaboração própria dos autores foi elaborado para captação dos seguintes dados: área/campo; linha de pesquisa; sistema do corpo humano acometido; contexto de coleta de dados; participantes da pesquisa; referencial teórico/base conceitual; referencial metodológico/técnica de análise; técnica de coleta de dados; tipo de abordagem; e principais resultados. Os dados foram categorizados tendo como base a Metodologia de Categorização Epistemológica para a Pesquisa na Enfermagem ${ }^{10}$, a qual está ancorada em conceitos da epistemologia bachelardiana e estrutura-se a partir de elementos de enfoque epistemológico.

Ressalta-se que os aspectos epistemológicos apresentados nesta pesquisa revelam não só os recursos utilizados pelos autores na geometrização de seus dados, como também a abstração dos mesmos no estudo do tema em tela. Esse fato é importante, pois valoriza as evidências epistemológicas trazidas pelos autores na perspectiva do pensamento científico de Bachelard ${ }^{11}$.

Por se tratar de um estudo documental cujas fontes são bancos de dados de domínio público, este artigo não precisou ser submetido à análise de um Comitê de Ética em Pesquisa. Todavia, primou-se pelo aspecto ético ao garantir a autoria das dissertações e teses selecionadas para análise, conforme Lei no 9.610/1998.

\section{RESULTADOS}

Os dados selecionados revelam que entre 2009 e 2019 dez dissertações de mestrado e duas teses de doutorado abordaram o tema criança com estoma. Os destaques epistemológicos das produções científicas selecionadas serão apresentados em três categorias: aspectos epistemológicos relacionados ao tema ou problema de pesquisa; aspectos epistemológicos relacionados ao enquadramento teórico-metodológico; e aspectos epistemológicos relacionados aos dados e/ou resultados.

\section{Aspectos epistemológicos relacionados ao tema ou problema de pesquisa}

Nesta categoria, apresentam-se as questões epistemológicas relacionadas ao tema ou problema de pesquisa. Consideraram-se o enquadramento dos estudos no esquema categorial (proposição gnoseológica) ${ }^{10}$ de agrupamento das pesquisas da Enfermagem (profissional, assistencial, organizacional), as linhas de pesquisa, sistema do corpo humano acometido pelo estoma, contexto de coleta de dados e os participantes da pesquisa. É uma categoria que apresenta em qual campo epistêmico do conhecimento (sujeito - imagem - objeto) os estudos estão predominantemente situados, como também os contornos epistemológicos na delimitação dos enfoques temáticos.

Identificou-se um predomínio de estudos na área/campo epistêmico assistencial com nove estudos, todos vinculados à linha de pesquisa: Processo de Cuidar em Saúde e Enfermagem. Três estudos estão situados na área/campo epistêmico profissional, todos vinculados à linha de pesquisa: Fundamentos Teórico-Filosóficos do Cuidar em Saúde e Enfermagem.

Quanto ao sistema orgânico acometido pelo estoma, observou-se que poucos estudos delimitaram a investigação para um tipo específico de estoma. Quando delimitado, identificou-se um interesse maior nos estomas que acometem o sistema digestório, com cinco estudos. Em um estudo, constatou-se que o estoma de interesse acometia o sistema respiratório. 
No que tange ao contexto de coleta de dados, notou-se um predomínio de estudos nos contextos: domiciliar e ambulatorial. Os familiares estiveram presentes como participantes de pesquisa em nove estudos. Também se observou estudos tendo como participantes de pesquisa a criança/adolescente, o professor de educação básica e os profissionais de saúde.

Na Figura 1, apresentam-se os aspectos epistemológicos relacionados ao tema ou problema de pesquisa.

\begin{tabular}{|c|c|c|c|c|c|}
\hline Código & $\begin{array}{l}\text { Área/campo } \\
\text { epistêmico }\end{array}$ & Linha de Pesquisa & $\begin{array}{c}\text { Sistema } \\
\text { acometido pelo } \\
\text { estoma }\end{array}$ & $\begin{array}{c}\text { Contexto de } \\
\text { coleta de dados }\end{array}$ & $\begin{array}{l}\text { Participantes } \\
\text { de pesquisa }\end{array}$ \\
\hline 01 & Assistencial & $\begin{array}{l}\text { Processo de cuidar em saúde } \\
\text { e enfermagem }\end{array}$ & $\begin{array}{l}\text { Não } \\
\text { especificado }\end{array}$ & Domiciliar & Familiares \\
\hline 02 & Assistencial & $\begin{array}{l}\text { Processo de cuidar em saúde } \\
\text { e enfermagem }\end{array}$ & $\begin{array}{l}\text { Sistema } \\
\text { digestório }\end{array}$ & $\begin{array}{l}\text { Hospitalar/ } \\
\text { ambulatorial }\end{array}$ & $\begin{array}{l}\text { Familiares/ } \\
\text { enfermeiros }\end{array}$ \\
\hline 03 & Assistencial & $\begin{array}{l}\text { Processo de cuidar em saúde } \\
\text { e enfermagem }\end{array}$ & $\begin{array}{l}\text { Não } \\
\text { especificado }\end{array}$ & Ambulatorial & crianças \\
\hline 04 & Assistencial & $\begin{array}{l}\text { Processo de cuidar em saúde } \\
\text { e enfermagem }\end{array}$ & $\begin{array}{l}\text { Não } \\
\text { especificado }\end{array}$ & $\begin{array}{l}\text { Hospitalar/ } \\
\text { ambulatorial }\end{array}$ & Familiares \\
\hline 05 & Assistencial & $\begin{array}{l}\text { Processo de cuidar em saúde } \\
\text { e enfermagem }\end{array}$ & $\begin{array}{l}\text { Sistema } \\
\text { digestório }\end{array}$ & Ambulatorial & Familiares \\
\hline 06 & Profissional & $\begin{array}{l}\text { Fundamentos Teórico- } \\
\text { Filosóficos do Cuidar em } \\
\text { Saúde e Enfermagem }\end{array}$ & $\begin{array}{l}\text { Sistema } \\
\text { respiratório }\end{array}$ & Domiciliar & Familiares \\
\hline 07 & Profissional & $\begin{array}{l}\text { Fundamentos Teórico- } \\
\text { Filosóficos do Cuidar em } \\
\text { Saúde e Enfermagem }\end{array}$ & $\begin{array}{l}\text { Sistema } \\
\text { digestório }\end{array}$ & Domiciliar & Familiares \\
\hline 08 & Assistencial & $\begin{array}{l}\text { Processo de cuidar em saúde } \\
\text { e enfermagem }\end{array}$ & $\begin{array}{l}\text { Não } \\
\text { especificado }\end{array}$ & $\begin{array}{l}\text { Domiciliar/ } \\
\text { escolar }\end{array}$ & $\begin{array}{l}\text { Familiares/ } \\
\text { Professores }\end{array}$ \\
\hline 09 & Assistencial & $\begin{array}{l}\text { Processo de cuidar em saúde } \\
\text { e enfermagem }\end{array}$ & $\begin{array}{l}\text { Não } \\
\text { especificado }\end{array}$ & Não se aplica & Não se aplica \\
\hline 10 & Assistencial & $\begin{array}{l}\text { Processo de cuidar em saúde } \\
\text { e enfermagem }\end{array}$ & $\begin{array}{l}\text { Não } \\
\text { especificado }\end{array}$ & Ambulatorial & $\begin{array}{l}\text { Criança/ } \\
\text { adolescente/ } \\
\text { familiares }\end{array}$ \\
\hline 11 & Profissional & $\begin{array}{l}\text { Fundamentos Teórico- } \\
\text { Filosóficos do Cuidar em } \\
\text { Saúde e Enfermagem }\end{array}$ & $\begin{array}{l}\text { Sistema } \\
\text { digestório }\end{array}$ & $\begin{array}{l}\text { Domiciliar/ } \\
\text { Hospitalar }\end{array}$ & $\begin{array}{l}\text { Cuidadores/ } \\
\text { profissionais de } \\
\text { saúde }\end{array}$ \\
\hline 12 & Assistencial & $\begin{array}{l}\text { Processo de cuidar em saúde } \\
\text { e enfermagem }\end{array}$ & $\begin{array}{l}\text { Sistema } \\
\text { digestório }\end{array}$ & $\begin{array}{l}\text { Domiciliar/ } \\
\text { Hospitalar }\end{array}$ & Familiares \\
\hline
\end{tabular}

Figura1: Aspectos epistemológicos relacionados ao tema ou problema de pesquisa. Rio de Janeiro, Brasil, 2020.

\section{Aspectos epistemológicos relacionados ao enquadramento teórico-metodológico}

Nesta categoria, apresentam-se as questões epistemológicas relacionadas ao referencial teórico ou base conceitual e ao referencial metodológico ou técnica de análise dos estudos. Conhecer o enquadramento teóricometodológico das dissertações e teses que versam sobre a criança com estoma possibilita compreender as diversas lentes e estruturas de análises sob as quais está calcada a epistemologia do tema.

Entre os referenciais teóricos utilizados pelos pesquisadores, estão Interacionismo Simbólico, Teoria da Probabilidade/Inferências Matemáticas, Aprendizagem Social de Vigotsky e Antropologia Interpretativa de Geertz. Entre as bases conceituais utilizadas, estão Cuidado de enfermagem na perspectiva de Colliére, Cuidado Centrado na Família e Criança, Família, Hospitalização e Brinquedo Terapêutico. Entre os métodos de análise, empregaram-se os seguintes: Teoria Fundamentada nos Dados, Revisão Sistemática e Método Etnográfico. Entre as técnicas de análise, estão Análise de Conteúdo do tipo Temática, Análise Estatística e Análise Narrativa.

Na Figura 2, apresentam-se os aspectos epistemológicos relacionados ao enquadramento teórico-metodológico. 


\begin{tabular}{cll}
\hline Código & \multicolumn{1}{c}{ Referencial Teórico/Base Conceitual } & \multicolumn{1}{c}{$\begin{array}{c}\text { Referencial Metodológico/ } \\
\text { Técnica de Análise }\end{array}$} \\
\hline 01 & Antropologia Interpretativa de Geertz & Método Etnográfico \\
02 & Não especificou & Análise de Conteúdo \\
03 & Teoria da Probabilidade/Inferências Matemáticas & Análise Estatística \\
04 & Teoria da Probabilidade/Inferências Matemáticas & Análise Estatística \\
05 & Interacionismo Simbólico & Análise Narrativa \\
06 & Cuidado de enfermagem na perspectiva de Colliére & Análise de Conteúdo \\
07 & Aprendizagem Social de Vigotsky/ Cuidado Centrado na Família & Análise de Discurso Francesa \\
08 & Não especificou & Análise Temática \\
09 & Criança, Família, Hospitalização e Brinquedo Terapêutico & Revisão Sistemática \\
10 & Teoria da Probabilidade/Inferências Matemáticas & Análise Estatística \\
11 & Teoria da Probabilidade/Inferências Matemáticas & Análise de Conteúdo/Análise Estatística \\
12 & Interacionismo Simbólico & Teoria Fundamentada nos Dados \\
\hline
\end{tabular}

FIGURA 2: Aspectos epistemológicos relacionados ao enquadramento teórico-metodológico. Rio de Janeiro, Brasil, 2020.

\section{Aspectos epistemológicos relacionados aos dados e resultados}

Nesta categoria, apresentam-se as questões epistemológicas relacionadas à coleta de dados, ao tipo de abordagem e aos principais resultados dos estudos selecionados. A esse respeito, observou-se um predomínio de estudos de abordagem qualitativa, tendo a entrevista como principal técnica de coleta de dados. Os principais resultados dos estudos são apresentados na Figura 3, o qual também revela aspectos epistemológicos relacionados aos dados.

\section{DISCUSSÃO}

A análise das dissertações e teses defendidas nos cursos de Pós-Graduação Stricto Sensu em Enfermagem, no Brasil, permitiu destacar questões epistemológicas relativas ao tema criança com estoma. Dos 12 estudos selecionados, dez foram desenvolvidos no Mestrado Acadêmico em Enfermagem e dois, no Doutorado Acadêmico em Enfermagem.

Ressalta-se que as produções científicas de enfermagem estão aglutinadas em linhas de pesquisa, organizadas em três áreas/campos epistêmicos: profissional, na qual estão as linhas de pesquisa vinculadas ao progresso da profissão, concentrando conteúdos e experiências mais substantivas do conhecimento de enfermagem; assistencial, na qual estão as linhas de pesquisa vinculadas aos efeitos do cuidado de enfermagem à clientela; e organizacional, na qual estão as linhas de pesquisa vinculadas aos modelos de organização e funcionamento da enfermagem nos serviços de saúde, mediante arranjos metodológicos e pedagógicos ${ }^{12}$.

Sobre o exposto, os resultados deste estudo apresentam uma concentração das produções científicas selecionadas na área/campo epistêmico assistencial, na linha de pesquisa: Processo de Cuidar em Saúde e Enfermagem, como também se destaca a escassez de estudos sobre criança com estoma na área/campo epistêmico organizacional.

Em geral, os estudos selecionados apresentaram uma tendência em não delimitar um tipo específico de estoma. No entanto, quando especificado, observou-se que os estomas que acometem o sistema digestório são os mais investigados, seguido daqueles que acometem o sistema respiratório.

A esse respeito, uma pesquisa realizada em um Hospital Público de Teresina, Piauí, constatou que, em relação ao tipo de estoma na criança, 84,74\% ( $n=50)$ acometeram o sistema digestório (33 colostomias, 11 gastrostomias, cinco ileostomias e uma esofagostomia), 13,55\% ( $n=8)$ o sistema respiratório (traqueostomia) e 11,86\% ( $n=7)$ o sistema urinário (cistostomia) ${ }^{6}$.

De modo semelhante, outro estudo realizado em um Hospital Público no Distrito Federal evidenciou que 68\% $(n=39)$ de crianças e adolescentes apresentavam colostomia e $23 \%(n=13)$ ileostomias ${ }^{13}$. Acerca dos estomas intestinais pediátricos, um estudo ${ }^{14}$ epidemiológico revelou que $88,2 \%$ das crianças e adolescentes hospitalizados em uma instituição de referência no Maranhão apresentavam colostomias e 11,8\% ileostomias, todos estomas temporários. Entre as causas, constam no citado estudo trauma (42,7\%), anomalias congênitas $(38,2 \%)$, causas obstrutivas $(5,4 \%)$ e inflamatórias, (4,5\%).

Quanto aos estomas que acometem o sistema respiratório, a traqueostomia destaca-se como a mais frequente. Estudo realizado com crianças traqueostomizadas no Sul do Brasil, entre janeiro de 2006 e novembro de 2015, identificou que a taxa de mortalidade foi de 31\%, considerada alta. Nesse mesmo estudo, a glossoptose foi a indicação mais comum para confecção da traqueostomia (30\%), seguida por estenose subglótica (16\%) e faringomalácia (11\%) 


\begin{tabular}{cccc}
\hline Código & $\begin{array}{c}\text { Tipo de } \\
\text { abordagem }\end{array}$ & $\begin{array}{c}\text { Técnica de coleta } \\
\text { de dados }\end{array}$ & \multicolumn{1}{c}{ Principais resultados } \\
\hline 01 & Qualitativa & $\begin{array}{l}\text { Entrevista e observação } \\
\text { participante }\end{array}$ & $\begin{array}{l}\text { A doença apresenta-se para a família como reformuladora de papéis. Há } \\
\text { divisão de tarefas dos pais relacionadas à higiene, troca de equipamento } \\
\text { coletor, alimentação e vida escolar. A comunicação com os pais deve ser } \\
\text { repensada. }\end{array}$ \\
& & & $\begin{array}{l}\text { A } \\
\text { A }\end{array}$
\end{tabular}

Qualitativa Entrevista e consulta ao prontuário

Quantitativa Consulta ao prontuário e observação direta observação participante; dinâmica de criatividade e sensibilidade bibliográfico Entrevista bibliográfico

12 Qualitativo Entrevista e observação participante

A gastrostomia se revelou como o mais frequente tipo de estoma do trato gastrintestinal das crianças no contexto investigado, sendo confeccionada sobremaneira por distúrbio de deglutição por neuropatia. A criança com estoma solicita uma atenção especial dos profissionais de saúde, sobremodo do enfermeiro.

A complicação mais frequente nos estomas na criança foi a dermatite de contato. Fatores de riscos identificados: idade, má adaptação ou não utilização do coletor e a ausência de demarcação do local de estoma.

As malformações congênitas $(69,1 \%)$ se apresentaram como as principais indicações de confecção do estoma. O sistema gastrintestinal foi o mais acometido (68\%). Houve predomínio de estomas temporários (43,6\%). A saída acidental da sonda de gastrostomia foi a complicação mais comum (50\%).

Observa-se uma aceitação progressiva dos pais às estomias gastrointestinais. Medo, insegurança e negação são sentimentos iniciais diante da necessidade de fazer a estomia. Observa-se sobrecarga materna e uma redução da rede de apoio.

Desafios da traqueostomia estão relacionados a nova condição, manejo da traqueostomia, falta de estrutura para o desenvolvimento do cuidado, presença frequente de secreção traqueal, receio com obstrução da cânula e descanulação.

Os principais locais de aprendizagem dos familiares cuidadores foram: ambulatório, hospital, domicílio. Os familiares cuidadores vivenciam desafios de ordem subjetiva, social, econômica na prática do cuidar da criança.

Observa-se o pouco conhecimento e despreparo dos familiares para lidar com a criança com estomia. Os familiares narraram apoio recebido principalmente da equipe de enfermagem. Os professores revelaram a importância do enfermeiro no contexto escolar.

Entre as evidências identificadas em defesa do uso do brinquedo terapêutico, estão: altos níveis de satisfação dos participantes e familiares e/ou profissionais; aumento da capacidade de a criança lidar de forma eficaz com sua condição clínica; redução de estresse, angústia e ansiedade da criança; adesão ao tratamento; motivação da criança para entender sobre sua situação devido à utilização de atividade lúdica; melhoria do bem-estar da criança; facilitação do autocuidado; promoção de maior independência; aplicabilidade da abordagem em qualquer nível de desenvolvimento; e a oportunidade de avaliação do nível atual de conhecimento da criança e de sua disposição para enfrentamento da situação clínica.

Crianças e adolescentes estomizados externaram independência, isolamento social, acometimento emocional e dificuldade em aceitar o tratamento. Por outro lado, seus pais identificaram a dependência dos filhos e não perceberam a exclusão social decorrente da superproteção.

A cartilha educativa mostrou-se um material educativo válido, podendo ser utilizada para auxiliar o cuidador no cuidado mais eficaz à criança com gastrostomia.

Para o casal, a experiência apresenta um duplo desafio: o de aprender a ser pais nessa condição e precisar prover um cuidado especializado para o seu filho. O cuidado especializado é um evento novo para os pais que passam a se sentir fragilizados por não possuírem uma referência social para basear suas interações. 
Independente da causa e do sistema orgânico afetado pelo estoma, cumpre ressaltar a necessidade de um planejamento de cuidado que contemple a prevenção de complicações imediatas e tardias, o adequado manejo do estoma pelos pais e/ou demais cuidadores, bem como um plano de alta que atenda às reais necessidades das crianças e dos seus familiares ${ }^{16-18}$.

Notou-se nos resultados que os contextos, domiciliar e ambulatorial, foram os mais investigados, como também um predomínio do familiar como participante de pesquisa. Esse achado pode estar associado ao fato de no contexto extra-hospitalar o familiar apresenta-se como principal cuidador da criança com estoma, sendo principalmente o pai e/ou a mãe, o que pode causar impactos na dinâmica e estrutura familiar.

Sobre o exposto, quase metade dos cuidadores de crianças com estoma em Uganda afirmou que seu conjugue deixou sua família e $93 \%$ dos cuidadores tiveram que deixar o trabalho para cuidar da criança em casa. Nesse mesmo estudo, $86 \%$ dos cuidadores eram mães, enquanto $13 \%$ eram pais ${ }^{19}$. Ademais, familiares de crianças com gastrostomia tendem a diminuir ou evitar saídas, enfrentam dificuldades no transporte, na obtenção de materiais necessários e financeiras, bem como preconceito e abandono de trabalho, culminando em possível sobrecarga e desequilíbrio familiar ${ }^{20}$.

Sabe-se que a escolha de um referencial teórico e metodológico deve ocorrer mediante uma consciência epistemológica coerente com o objeto e objetivo(s) do estudo. Entre os referenciais teóricos utilizados nos estudos selecionados, estão Aprendizagem Social de Vigotsky, a Antropologia Interpretativa de Geertz, o Interacionismo Simbólico e as Inferências Matemáticas/Teoria da Probabilidade. Também foram identificadas nos estudos bases conceituais relacionadas ao Cuidado na perspectiva de Colliére; Cuidado Centrado na Família; Criança, Família, Hospitalização e Brinquedo Terapêutico.

Estudo realizado com o objetivo de analisar as dissertações e teses do programa de pós-graduação em Enfermagem de uma universidade federal do Nordeste brasileiro identificou que a Teoria das Necessidades Humanas Básicas foi utilizada em 37\% dos estudos, seguida da Teoria das Representações Sociais de Moscovici $(32,6 \%)^{21}$

No que tange aos referenciais metodológicos ou técnicas de análise encontrados nos estudos, estão Teoria Fundamentada nos Dados, Método Etnográfico, Análise de conteúdo, Análise Narrativa, Revisão Sistemática e Análise Estatística. Ressalta-se uma predominância de métodos de pesquisa que favorecem a compreensão dos fenômenos de pesquisas.

De igual modo, estudo desenvolvido a fim de analisar a formação de doutores no Brasil identificou que, no triênio 2010-2012, a abordagem predominante nas teses de doutorado foi a qualitativa (330-66,1\%), seguida da quantitativa $(139-27,9 \%)$ e qualiquantitativa $(30-6,0 \%)^{22}$. No tocante à abordagem qualitativa, compreende-se que o pesquisador quando a utiliza no campo da saúde trabalha com uma infinidade de informações, as quais necessitam ser organizadas e analisadas para alcançar a compreensão do fenômeno, exigindo do mesmo reflexividade e posicionalidade na produção do conhecimento ${ }^{23}$.

Nos estudos de abordagem qualitativa selecionados, constatou-se que a entrevista foi a técnica de coleta de dados mais empregada pelos pesquisadores. A partir da entrevista, é possível uma imersão nos dados, que são obtidos mediante uma interação dialógica entre o entrevistador e o entrevistado ${ }^{24}$. A realização de uma entrevista possibilita ao pesquisador um aprofundamento das questões em estudo por meio da interação face a face, retomada de pontos não bem esclarecidos e percepção de gestos e fisionomia dos participantes ${ }^{25}$. Portanto, a entrevista apresenta-se como uma técnica de coleta de dados muito apropriada para os estudos de abordagem qualitativa.

\section{CONCLUSÃO}

Os resultados obtidos a partir desta pesquisa possibilitaram compreender que a produção do conhecimento relativa à criança com estoma nos cursos de Pós-Graduação Stricto Sensu em Enfermagem, no Brasil, está predominantemente pautada na epistemologia da compreensão que envolve o cuidar e o cuidado à criança com estoma e sua família. Notou-se um interesse dos pesquisadores para a ação do cuidar, comprovado por uma maior concentração de estudos na área campo/epistêmico assistencial, na linha de pesquisa: Processo de Cuidar em Saúde e Enfermagem. Esse fato revela uma perspectiva pragmática dos pesquisadores sobre o tema em tela.

Há uma tendência das pesquisas para o contexto domiciliar e ambulatorial, sendo os familiares os participantes de pesquisa mais investigados. Este estudo tem como limitação a análise de pesquisas provenientes apenas de cursos de Pós-Graduação Stricto Sensu em Enfermagem. Assim, aponta-se como direcionamento o aprofundamento dessa análise para os cursos de Pós-Graduação Stricto Sensu e Lato Sensu de áreas afins ao campo de saber da Enfermagem.

\section{REFERÊNCIAS}

1. Associação Brasileira de Estomaterapia (SOBEST). Diretrizes éticas para o exercício da Estomaterapia (online). 2016 [cited 2020 Jan 03]. Available from: http://www.sobest.org.br/arquivos/codigo-de-etica-sobest.pdf 
2. Dias MSC, Paula MAB, Morita ABPS. Perfil profissional de enfermeiros estomaterapeutas egressos da universidade de Taubaté. ESTIMA - Bras J Enterestomal Ther (online). 2014 [cited 2020 Jan 03]; 12(3). Available from: https://www.revistaestima.com.br/index.php/estima/article/view/92

3. Teixeira AKS, Menezes LCG, Oliveira RM. The View of Nursing Managers about a Stomatherapy Service in a Public Reference Hospital. ESTIMA - Bras J Enterestomal Ther (online). 2016 [cited 2020 Jan 03]; 14(1):3-12. DOI: https://doi.org/10.5327/Z18063144201600010002

4. Santos VLCG, Cesaretti IUR. Assistência em Estomaterapia: cuidando de pessoas com estomia. São Paulo (SP): Editora Atheneu; 2015.

5. Dias SM, Gonçalves FG. Percepções de familiares com criança estomizada sobre o grupo de apoio. ESTIMA - Bras J EnterestomalTher(online). 2015 [cited 2020 Jan 03]; 13(2). Available from: https://www.revistaestima.com.br/index.php/estima/article/view/227

6. Costa ECL, Vale DS, Luz MHBA. Profi le of Stomized Children in a Public Hospital of Teresina, Piauí, Brazil. ESTIMA - Bras J EnterestomalTher(online). 2016 [cited 2020 Jan 03]; 14(4):169-174. DOI: https://doi.org/10.5327/Z1806-3144201600040003

7. Associação Brasileira de Estomaterapia (SOBEST). Cursoscredenciados (online). 2018 [cited 2020 Jan 03]. Available from: http://www.sobest.org.br/arquivos/CURSOS_ATUALIZADOS_NOV_2018.pdf

8. Correia AKS, Moreira TMM, Castro ME, Lopes CHAF, Jorge MSB. Analysis of nursing dissertations and thesis on ostomy wounds, brazil, 1979-2005. Rev Rene (online). 2008 [cited 2020 Jan 03]; 9(2):106-112. Available from: http://www.periodicos.ufc.br/rene/article/view/5045/3705

9. Melo MC, Kamada I. O papel da família no cuidado à criança com estoma intestinal: uma revisão narrativa. ESTIMA - Bras J EnterestomalTher(online). 2015 [cited 2020 Jan 03];13(3). Available from: https://www.revistaestima.com.br/index.php/estima/article/view/219

10. Carvalho V, Figueiredo NMA, Leite JL, Moreira MC. Epistemological questions on the construction of knowledge in nursing: from teaching to the practice of caring. Esc. Anna Nery Rev. Enferm. (online). 2003 [cited 2020 Jan 03]; 7(2):156-166. Available from: https://pesquisa.bvsalud.org/portal/resource/pt/lil-354004

11. Bachelard G. A formação do espírito científico. Rio de Janeiro: Contraponto; 1996.

12. Carvalho V. Research lines in nursing: phylosophical and epistemological highlights. Rev. bras. enferm (online). 2015 [cited 2020 Jan 03]; 68(4):723-9. DOI: http://dx.doi.org/10.1590/0034-7167.2015680421p

13. Monteiro SNC, Kamada I, Silva AL, Souza TCR. Perfil de crianças e adolescentes estomizados atendidos em um Hospital Público do Distrito Federal. ESTIMA - Bras J EnterestomalTher (online). 2014 [cited 2020 Jan 03]; 12(3). Available from: https://www.revistaestima.com.br/index.php/estima/article/view/93

14. Santos OJ, Filho ENS, Filho AKDB, Desterro VS, Silva MVT, Prado RPS, et al. Children and adolescentesostomized in a reference hospital. Epidemiological profile. J. coloproctol (online). 2016 [cited 2020 Jan 03]; 36(2):75-9. DOI: http://dx.doi.org/10.1016/j.jcol.2016.03.005

15. Schweiger C, Manica D, Becker CF, Abreu LSP, Manzini M, Sekine L, et al. Traqueostomy in children: a ten-year experience from a tertiary center in southern Brazil. Braz. j. otorhinolaryngol (online). 2017 [cited 2020 Jan 03]; 83(6):627-32. DOI: https://doi.org/10.1016/j.bjorl.2016.08.002

16. Krishnamurty DM, Blatnik J, Much M. Stoma complications. Clin Colon Rectal Surg (online). 2017 [cited 2020 Jan 03]; 30(3):193200. DOI: https://doi.org/10.1055/s-0037-1598160

17. Smith J, Cheater F, Bekker H. Parent's experiences of living with a child with long-term condition: a rapid structed review of the literature. Health expect (online). 2013 [cited 2020 Jan 03]; 18(4):452-474. DOI: https://doi.org/10.1111/hex.12040

18. Prinz A, Colwell JC, Cross HH, Mantel J, Perkins J, Walker CA. Discharge planning for a patient with new ostoma. J Wound Ostomy Continence Nurs (online). 2015 [cited 2020 Jan 03]; 42(1):79-82. DOI: https://doi.org/10.1097/WON.0000000000000094

19. Muzira A, Kakembo N, Kisa P, Langer M, Sekabira J, Ozgediz D, et al. The socioeconomic impact of a pediatric ostomy in Uganda; a pilot study. Pediatr. surg. int [Internet]. 2018 [citado em 2020 jan 03]; 34(4):457-66. DOI: https://doi.org/10.1007/s00383018-4230-8

20. Mela CS, Zacarin CFL, Dupas G. Family evaluation of children and adolescents submitted to gastromy. Rev. eletrônica enferm. (online). 2015 [cited 2020 Jan 03]; 17(2):212-22. DOI: http://dx.doi.org/10.5216/ree.v17i2.29049

21. Pimenta CJL, Fernandes WAAB, Falcão RMM, Freitas AS, Oliveira JS, Costa KNFM. Analysis of the dissertations and theses of the Graduate Nursing Program of Universidade Federal da Paraíba. Rev. Min. Enferm. (online). 2018 [cited 2020 Jan 03]; 22(e):1093. DOI: https://www.dx.doi.org/10.5935/1415-2762.20180023

22. Scochi CGS, Gelbcke FL, Ferreira MA, Lima MADS, Padilha KG, Padovani NA, et al. Nursing doctorates in Brazil: research formation and theses production. Rev. latinoam. enferm. (online). 2015 [cited 2020 Jan 03]; 23(3):387-94. DOI: https://doi.org/10.1590/0104-1169.0590.2564

23. Silva A, Castro-Silva CR, Moura L. Qualitative research in health: routes and difficulties in beginner researchers' education. Saude Soc. (online). 2018 [cited 2020 Jan 03]; 27(2):632-45. DOI: http://dx.doi.org/10.1590/s0104-12902018172700

24. Gomes IM, Lacerda MR, Rodrigues JAP, Nascimento JD, Camargo TB. Use of external interviewers in qualitative research: action plan. Enferm. glob. (online). 2016 [cited 2020 Jan 03]; 15(4):235-63. DOI: https://doi.org/10.6018/eglobal.15.4.240091

25. Tabosa HR, Pinto VB, Loureiro JMM. Análise de regularidades metodológicas em pesquisas brasileiras sobre comportamentos de uso e usuários da informação. InvestigBibl (online). 2016 [cited 2020 Jan 03]; 30(70):249-67. DOI:

https://doi.org/10.1016/j.ibbai.2016.10.011 